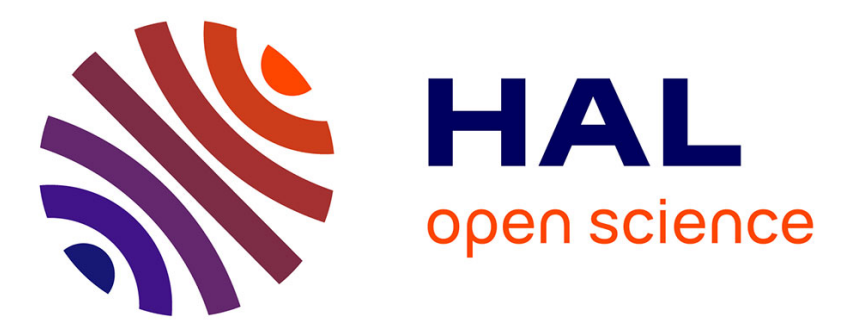

\title{
Sparse reconstruction from Multiple-Angle Total Internal Reflection Fluorescence Microscopy
}

Emmanuel Soubies, Laure Blanc-Féraud, Sébastien Schaub, Gilles Aubert

\section{To cite this version:}

Emmanuel Soubies, Laure Blanc-Féraud, Sébastien Schaub, Gilles Aubert. Sparse reconstruction from Multiple-Angle Total Internal Reflection Fluorescence Microscopy. ICIP - International Conference on Image Processing, Oct 2014, Paris, France. pp.2844 - 2848, 10.1109/ICIP.2014.7025575 . hal01037895

\section{HAL Id: hal-01037895 \\ https://hal.inria.fr/hal-01037895}

Submitted on 23 Jul 2014

HAL is a multi-disciplinary open access archive for the deposit and dissemination of scientific research documents, whether they are published or not. The documents may come from teaching and research institutions in France or abroad, or from public or private research centers.
L'archive ouverte pluridisciplinaire HAL, est destinée au dépôt et à la diffusion de documents scientifiques de niveau recherche, publiés ou non, émanant des établissements d'enseignement et de recherche français ou étrangers, des laboratoires publics ou privés. 


\title{
SPARSE RECONSTRUCTION FROM MULTIPLE-ANGLE TOTAL INTERNAL REFLECTION FLUORESCENCE MICROSCOPY
}

\author{
Emmanuel Soubies $^{\star}$, Laure Blanc-Féraud ${ }^{\star}$, Sébastien Schaub ${ }^{\dagger}$ and Gilles Aubert ${ }^{\ddagger}$ \\ * Université Nice Sophia Antipolis, I3S, CNRS, Sophia Antipolis, France \\ † Université Nice Sophia Antipolis, iBV, CNRS, INSERM, Nice, France \\ $\ddagger$ Université Nice Sophia Antipolis, Laboratoire J.A. Dieudonné, CNRS, Nice, France
}

\begin{abstract}
Super-resolution microscopy techniques allow to overstep the diffraction limit of conventional optics. Theses techniques are very promising since they give access to the visualisation of finer structures which is of fundamental importance in biology. In this paper we deal with Multiple-Angle Total Internal Reflection Microscopy (MA-TIRFM) which allows to reconstruct 3D sub-cellular structures of a single layer of $\sim 300 \mathrm{~nm}$ behind the glass coverslip with a high axial resolution. The $3 \mathrm{D}$ volume reconstruction from a set of $2 \mathrm{D}$ measurements is an ill-posed inverse problem and a regularization is essential. Our aim in this work is to propose a new reconstruction method for sparse structures robust to Poisson noise and background fluorescence. The sparse property of the solution can be seen as a regularization using the ' $\ell^{0}$ norm'. In order to solve this combinatorial problem, we propose a new algorithm based on smoothed ' $\ell^{0}$ norm' allowing minimizing a non convex energy, composed of the Kullback-Leibler divergence data term and the $\ell^{0}$ regularization term, in a Graduated Non Convexity framework.
\end{abstract}

Index Terms - Inverse problems, Image reconstruction, Highresolution imaging, Evanescent wave microscopy

\section{INTRODUCTION}

Understanding tumor organisation and development is a major challenge in current biological researches. Super-resolution microscopy techniques are widely used in this context since they allow the visualisation of small sub-cellular structures near the cell membrane. Indeed, these new and powerful tools allow to overstep the limitations of conventional optics for which the axial resolution is restricted to a few hundred of nanometers (e.g. $\sim 500 \mathrm{~nm}$ for confocal microscope).

In this study we deal with Total Internal Reflection Fluorescence Microscopy (TIRFM) [1] which gives access (in the better configuration) to a single layer of $\sim 100 \mathrm{~nm}$ behind the glass coverslip $(5 \times$ better, in terms of axial resolution, than confocal microscopy) and provides low background fluorescence and high signal-to-noise ratio images. While single TIRFM acquisition is actually a unique projection (2D image) of the observed volume and can not provide 3D information, Multiple-Angle TIRFM (MA-TIRFM) provides a set of TIRFM acquisitions at different incident angles. Through the development of dedicated algorithms, these acquisitions allow to reconstruct the 3D volume of the sample and overcome the axial resolution of single TIRFM. Indeed the set of 2D images obtained from

Contact: soubies@i3s.unice.fr,_blancf@i3s.unice.fr, sebastien.schaub@unice.fr, gilles.aubert@unice.fr
MA-TIRFM corresponds to different projections (with different integration depth) of the 3D volume on the $(x, y)$ plane. In the following we study the ill-posed inverse problem of finding axial positions of the observed structures from this set of images. This is a reconstruction problem.

Some authors have proposed different methods to estimate the 3D sub-cellular volume from MA-TIRFM acquisitions. All of these methods use a strong shape prior on the unknown structures. In [2, 3] axial cell membrane position is estimated for each pixel of the $(x, y)$ plane using a simple one-dimensional geometry (top hat). Estimating position and diameter of isolated secretory granules have been studied in $[4,5]$ where granules are modelled by spherical or cubic volumes. A statistical framework for curvilinear structures reconstruction (microtubules) is proposed in [6]. More recently, 3D particle estimation is achieved in a MAP-Bayesian framework [7] and a Marked Point Process approach is used in [8] to deal with objects features estimation.

In this paper, we propose a new model without strong shape constraints on the observed structures. We formulate the reconstruction problem as a minimization of the sum of two terms ; a data fidelity term defined from the likelihood function of the observations given the solution, and, a regularization term. We focus particularly on finding sparse solutions to the problem since we are interested in reconstructing cell membranes and fine particles which are sparse structures in the medium. Moreover, sparse property could also be useful in the case where the solution is searched in a particular basis (e.g. in a wavelet basis). Sparsity of the solution can be modelled using the ' $\ell^{0}$ norm' ${ }^{1}$ as regularization term. Following recent work [9], this difficult combinatorial optimization problem is tackled by using the SL0 algorithm, based on smoothed ' $\ell^{0}$ norm' and using a Graduated Non Convexity (GNC) approach [10]. In [9], the SL0 algorithm is developed for finding sparse solutions of underdetermined system of linear equations. In the following we propose a new algorithm based on this principle allowing the minimization of a non convex energy defined by the sum of the Kullback-Leibler divergence data term adapted to Poisson noise and the $\ell^{0}$ regularization term enforcing sparsity of the solution.

The paper is organized as follows. Section 2 formalizes the TIRF image formation. We begin Section 3 with the formulation of the inverse problem as a minimization problem and we propose to solve this problem by minimizing a sequence of functional where the ' $\ell^{0}$ norm' is approximated using smooth functions. We show that we can define a sequence of functional which starts with a convex one (on a large convex set) and introduce progressively the non convexity of the ' $\ell^{0}$ norm'. Then we give a theoretical result on the limit of the minimizers with respect to the ' $\ell^{0}$ norm'. Finally, some

\footnotetext{
${ }^{1}$ We write ' $\ell 0$ norm' because the absolute scalability is not satisfied.
} 
numerical results are presented in Section 4.

\section{IMAGE FORMATION AND PROBLEM STATEMENT}

Total internal reflection of a light beam, at the interface between two mediums of refractive indices $n_{i}$ (incident) and $n_{t}$ (transmitted), occurs when the incident angle $\alpha$ of the light beam becomes greater than a critical angle $\alpha_{c}$. This phenomenon produces an evanescent wave capable of exciting fluorophores near the dielectric surface. Theoretically [1], the axial profile of the evanescent field can be modelled using a decreasing exponential depending on the incident angle. In the following, we denote by:

$$
\begin{aligned}
& \text { - } \mathscr{O}^{d}=\{1, \ldots, n\} \times\{1, \ldots, m\} \times\{1, \ldots, p\} \subset \mathbb{N}^{3} \text { the } \\
& \text { image space (3D) } \\
& \text { - } \mathscr{M}=\{1, \ldots, n\} \times\{1, \ldots, m\} \times\{1, \ldots, L\} \subset \mathbb{N}^{3} \text { the } \\
& \text { measures space }(2 \mathrm{D}+\text { angle) }
\end{aligned}
$$

where $n$ and $m$ are the images' size (in the $(x, y)$ plane) indexed in the following by $i \in\{1, \ldots, n\} \times\{1, \ldots, m\}, p$ is the number of voxels of the $3 \mathrm{D}$ unknown image in the axial direction (indexed by $j$ ) and $L$ the number of incident angles used (i.e number of acquired $2 \mathrm{D}$ images), indexed by $l$.

Let $\mathrm{f}: \mathscr{O}^{d} \longrightarrow \mathbb{R}$ denote the 3D unknown fluorophore density and $\mathrm{s}: \mathscr{M} \longrightarrow \mathbb{R}$ the set of recorded images. According to the model given in [1] and considering shot noise and background fluorescence degradations, TIRF image formation can be formulated as follows: $\forall(i, l) \in \mathscr{M}$,

$$
\mathrm{s}(i, l)=\mathscr{P}\left(\mathrm{H}\left(\mathrm{f}+b_{g}\right)(i, l)\right)
$$

where $\mathscr{P}$ denotes the Poisson distribution, which mimics the photon collection process, $b_{g}$ is a constant that models the background fluorescence produced by parasites light sources in the medium (e.g. autofluorescence, light reflection...) and $\mathrm{H}$ is the TIRF operator defined by: $\forall \mathrm{f} \in \ell^{2}\left(\mathscr{O}^{d}\right), \forall(i, l) \in \mathscr{M}$,

$$
\mathrm{H}(\mathrm{f})(i, l)=\sum_{j=1}^{p} \mathrm{f}(i, j) \exp \left(-\frac{z_{j}}{d_{l}}\right) \delta_{z}(j) \mu_{i} .
$$

Here, $\delta_{z}(j)$ is the $j^{t h}$ discretization step along the $(O z)$ axis, $\mu_{i}$ is the $i^{t h}$ pixel area (2D) and $d_{l}$ models the penetration depth of the evanescent wave corresponding to the incident angle $\alpha_{l}, l \in$ $\{1 \cdots L\}$.

Figure 1 contains an example of MA-TIRFM acquisitions from a sample of cortactin. It is readily seen from these images that when the penetration depth decreases (from left to right), some fluorophores are not excited anymore. These structures are the deeper ones and so we can get $3 \mathrm{D}$ information from this set of images.

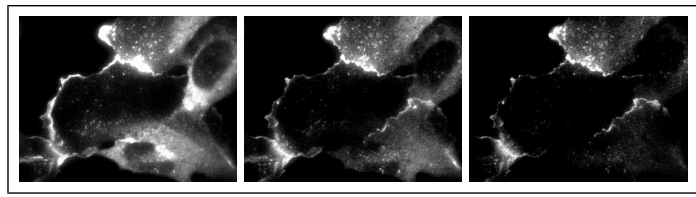

Fig. 1. Multiple-angle TIRFM (MA-TIRFM) acquisitions (2D images $\mathrm{s}(i, l),(i, l) \in \mathscr{M}$ ) from a cortactin sample (protein involved in cell adhesion). Left : deeper penetration depth $\left(\alpha \simeq \alpha_{c}\right)$. Right : thinner penetration depth $\left(\alpha \simeq \alpha_{\max }\right)$.

\section{SPARSE RECONSTRUCTION METHOD}

\subsection{Optimization Problem Formulation}

Finding a solution of the ill-posed inverse problem $(\mathcal{P})$ (i.e determine $\mathrm{f}$ in $(\mathcal{P})$ from acquisition $\mathrm{s}$ ) can be formulated as a minimization problem. Assuming that observations s given the fluorophore density $f$ are pairwise independent (which can be justified by the fact that for a given $\mathrm{f}$, data depend only to the noise which is pairwise independent), then maximizing the likelihood function of the observations $\mathrm{s}$ given $\mathrm{f}$ leads to the following problem:

$$
\tilde{\mathrm{f}}=\underset{\mathrm{f} \in \ell^{2}\left(\mathscr{O}^{d}\right)}{\arg \min } J_{d}(\mathrm{f})
$$

where $J_{d}$ is defined by ${ }^{2}$ :

$$
J_{d}(\mathrm{f})=\sum_{(i, l) \in \mathscr{M}}-\mathrm{s}(i, l) \log \left[\mathrm{H}\left(\mathrm{f}+b_{g}\right)(i, l)\right]+\mathrm{H}\left(\mathrm{f}+b_{g}\right)(i, l) .
$$

Let's give some properties of $J_{d}$ which will be used, in the following, to prove theoretical results:

- It is easy to show that $J_{d}$ is continuous and convex

- It has been proved in [11], in the case where $\mathrm{H}$ is a convolution operator, that:

$$
\exists a>0, b \in \mathbb{R} s . t \forall \mathrm{f} \in \ell^{2}\left(\mathscr{O}^{d}\right), J_{d}(\mathrm{f}) \geq a\|\mathrm{f}\|_{1}+b
$$

where $\|.\|_{1}$ is the $\ell^{1}$ norm. TIRF operator (1) satisfies the conditions used in [11] and then (3) holds which implies that $J_{d}$ is coercive.

Since we are interesting in finding a sparse solution of the problem, we will consider, in the following, the regularized problem:

$$
\hat{\mathrm{f}}=\underset{\mathrm{f} \in \ell^{2}\left(\mathscr{O}^{d}\right)}{\arg \min } G(\mathrm{f}):=J_{d}(\mathrm{f})+\lambda\|\mathrm{f}\|_{0}
$$

where $\|\mathrm{f}\|_{0}=\#\left\{(i, j) \in \mathscr{O}^{d} ; \mathrm{f}(i, j) \neq 0\right\}$ and $\lambda \in \mathbb{R}^{+}$is a weight parameter.

\subsection{SL0 Algorithm}

Solving problem $\left(\mathcal{P}_{2}\right)$ with a combinatorial search is NP-hard. However, it has been shown [9] that a smoothed ' $\ell^{0}$ norm' can be used for finding sparse solution of an undetermined system of linear equations. The main idea in [9] is to approximate the ' $\ell^{0}$ norm' by a suitable continuous function depending on a parameter $\sigma>0$. For example, they propose the following smooth approximation:

$$
\|\mathrm{f}\|_{0} \simeq J_{\sigma}(\mathrm{f}):=N-\sum_{(i, j) \in \mathscr{O}^{d}} h_{\sigma}(\mathrm{f}(i, j))
$$

where $N=\left|\mathscr{O}^{d}\right|$ is the number of voxels in $\mathrm{f}$ and $h_{\sigma}$ a continuous function defined $b^{3}{ }^{3}$ :

$$
h_{\sigma}(s)=\exp \left(-\frac{s^{2}}{2 \sigma^{2}}\right) .
$$

It is easy to show that $J_{\sigma}$ tends to the ' $\ell^{0}$ norm' when $\sigma$ tends to zero and consequently the smaller the values of $\sigma$ the better the

\footnotetext{
${ }^{2}$ Note that minimizing $J_{d}$ in (2) is equivalent to minimize the well known Kullback-Leibler divergence.

${ }^{3}$ In [9] they propose a general class of function for $h_{\sigma}$. In this paper we will only deal with the function $h_{\sigma}$ defined in (5).
} 
approximation. On the other side the larger the value of $\sigma$, the smoother the approximation. Following the idea in [9], we propose to solve a series of optimization problems:

$$
\mathrm{f}^{\sigma}=\underset{\mathrm{f} \in \ell^{2}\left(\mathscr{O}^{d}\right)}{\arg \min } G_{\sigma}(\mathrm{f}):=J_{d}(\mathrm{f})+\lambda J_{\sigma}(\mathrm{f})
$$

for a decreasing sequence of $\sigma$. At each step (i.e for each $\sigma$ of the sequence), problem $\left(\mathcal{P}_{2}^{\sigma}\right)$ is tackled using a descent algorithm ${ }^{4}$ initialized with the solution obtained for the previous (larger) value of $\sigma$. These iterates allow to introduce gradually the non-convexity of the problem and escape from local minima (GNC approach [10]). Let remark that our work differs from [9] in the sense that [9] focus on the resolution of linear systems under ' $\ell^{0}$ norm' sparsity condition.

We will show that a large enough value of $\sigma$ ensures that problem $\left(\mathcal{P}_{2}^{\sigma}\right)$ has no local minima. It can be easily shown that $J_{\sigma}$ is strictly convex on $[-\sigma, \sigma]^{N}$. Then the following property states:

Property 1. There exists a large enough value of $\sigma$ denoted $\sigma_{0}$ such that:

$$
\forall \sigma \geq \sigma_{0} \quad \forall(i, j) \in \mathscr{O}^{d},\left|\mathrm{f}^{\sigma}(i, j)\right| \leq \sigma
$$

where $\mathrm{f}^{\sigma}$ is a solution of $\left(\mathcal{P}_{2}^{\sigma}\right)$.

Proof. Let $\tilde{\mathrm{f}}$ be a solution of the problem $\left(\mathcal{P}_{1}\right)$ (i.e a solution of $\left(\mathcal{P}_{2}^{\sigma}\right)$ with $\lambda=0$ ). Such a solution exists since $J_{d}$ is continuous, convex and coercive. Because $\mathrm{f}^{\sigma}$ is a solution of $\left(\mathcal{P}_{2}^{\sigma}\right)$ and that $\forall \mathrm{f} \in$ $\ell^{2}\left(\mathscr{O}^{d}\right), J_{\sigma}(\mathrm{f}) \leq N$ we have:

$$
J_{d}\left(\mathrm{f}^{\sigma}\right)+\lambda J_{\sigma}\left(\mathrm{f}^{\sigma}\right) \leq J_{d}(\tilde{\mathrm{f}})+\lambda J_{\sigma}(\tilde{\mathrm{f}}) \leq J_{d}(\tilde{\mathrm{f}})+\lambda N .
$$

Using result (3) and the fact that $\forall \mathrm{f} \in \ell^{2}\left(\mathscr{O}^{d}\right), J_{\sigma}(\mathrm{f}) \geq 0$ we have: $\exists a>0, b \in \mathbb{R}$ such that,

$$
a\left\|\mathrm{f}^{\sigma}\right\|_{1}+b \leq J_{d}\left(\mathrm{f}^{\sigma}\right) \leq J_{d}\left(\mathrm{f}^{\sigma}\right)+\lambda J_{\sigma}\left(\mathrm{f}^{\sigma}\right) \leq J_{d}(\tilde{\mathrm{f}})+\lambda N .
$$

Consequently, $\forall(i, j) \in \mathscr{O}^{d},\left|\mathrm{f}^{\sigma}(i, j)\right|$ is bounded by a value independent of $\sigma$ and then by taking $\sigma_{0}$ equal to this bound, equation (6) is satisfied.

From Property 1, we ensure that by taking a large enough value of $\sigma_{1}$ to initialize the algorithm, the solution $\mathrm{f}^{\sigma_{1}}$ of the approximated problem will be in the convex part of $J_{\sigma}$ (i.e $\mathrm{f}^{\sigma_{1}} \in\left[-\sigma_{1}, \sigma_{1}\right]^{N}$ ) which ensures $\mathrm{f}^{\sigma_{1}}$ to be unique since $J_{\sigma}$ is strictly convex on $[-\sigma, \sigma]^{N}$. Finally the proposed algorithm is described in Algo 1.

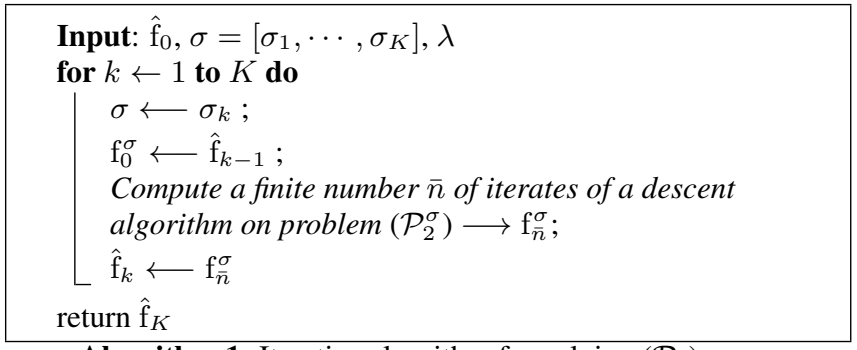

Algorithm 1: Iterative algorithm for solving $\left(\mathcal{P}_{2}\right)$

The decrease of the sequence of $\sigma$ is chosen such as $\sigma_{k}=$ $c \sigma_{k-1}, k \geq 2$ where $c$ is a coefficient between 0.5 and 1 . The first value, $\sigma_{1}$ is chosen according to Property 1 in order, as explained before, to ensure the uniqueness of the minimizer of $\left(\mathcal{P}_{2}^{\sigma}\right)$. We stop the algorithm when $\sigma$ becomes smaller than the estimated $b_{g}$ (the $b_{g}$

${ }^{4}$ For example the regularized Richardson-Lucy algorithm [12]. constant is estimated from an acquired image region, selected manually, without biological structures). Note that for each $\sigma$ in Algorithm 1 , only a finite number $\bar{n}$ of a descent algorithm iterates are performed which is sufficient. In practice it is not necessary to converge to the minimiser of problem $\left(\mathcal{P}_{2}^{\sigma}\right)$ for each $\sigma$. In this paper we use the regularized Richardson-Lucy algorithm [12] to perform the descent step. Finally, we initialize $\hat{\mathrm{f}}_{0}$ by a uniform $3 \mathrm{D}$ image equal to the estimated value of $b_{g}$.

\subsection{A Theoretical Result on the Limit of $\left(\mathcal{P}_{2}^{\sigma}\right)$}

The following theorem ensures that the global minimizers of $\left(\mathcal{P}_{2}^{\sigma}\right)$ are also global minimizers of $\left(\mathcal{P}_{2}\right)$ when $\sigma$ tends to zero.

Theorem 1. Let $\mathcal{S}_{0}$ be the set of global solutions of problem $\left(\mathcal{P}_{2}\right)$ and $\mathcal{S}_{\sigma}$ the one of problem $\left(\mathcal{P}_{2}^{\sigma}\right)$, then:

$$
\lim _{\sigma \rightarrow 0} \mathcal{S}_{\sigma} \subseteq \mathcal{S}_{0}
$$

in the sense of Painleve-Kuratowski convergence [13].

Proof. It is easy to prove the existence of a solution of $\left(\mathcal{P}_{2}\right)$ and $\left(\mathcal{P}_{2}^{\sigma}\right)$ which implies that $\mathcal{S}_{0}$ and $\mathcal{S}_{\sigma}$ are a non-empty sets. We define by $\mathscr{E}_{\gamma}\left(J_{d}\right):=\left\{\mathrm{f}\right.$ s.t $\left.J_{d}(\mathrm{f}) \leq \gamma\right\}$ the $\gamma$ sublevel set of $J_{d}$. Let $\hat{\mathrm{f}} \in \mathcal{S}_{0}$ and $\mathrm{f}^{\sigma} \in \mathcal{S}_{\sigma}$, then we have the following inequality:

$$
\forall \sigma, G_{\sigma}\left(\mathrm{f}^{\sigma}\right) \leq G_{\sigma}(\hat{\mathrm{f}}) \leq G(\hat{\mathrm{f}}) .
$$

The first inequality comes from the fact that $\mathrm{f}^{\sigma}$ is a minimizer of $\left(\mathcal{P}_{2}^{\sigma}\right)$ and the second inequality is true since $\forall \sigma, \forall \mathrm{f} \in \ell^{2}\left(\mathscr{O}^{d}\right), J_{\sigma}(\mathrm{f}) \leq$ $\|\mathrm{f}\|_{0}$. From (10) we get:

$$
\forall \sigma, \mathrm{f}^{\sigma} \in \mathcal{S}_{\sigma} \subseteq \mathscr{E}_{G(\hat{\mathrm{f}})}\left(G_{\sigma}\right) .
$$

Now, we show that:

$$
\mathscr{E}_{G(\hat{\mathrm{f}})}\left(G_{\sigma}\right) \underset{\sigma \rightarrow 0}{\longrightarrow} \mathscr{E}_{G(\hat{\mathrm{f}})}(G)=\mathcal{S}_{0}
$$

which is equivalent (in the sense of Painleve-Kuratowski convergence [13]) to satisfy the two following conditions:

$$
\begin{aligned}
& \forall \mathrm{f} \in \mathscr{E}_{G(\hat{\mathrm{f}})}(G), \exists \sigma_{0}>0, \forall \sigma>0, \sigma \leq \sigma_{0} \Rightarrow \mathrm{f} \in \mathscr{E}_{G(\hat{\mathrm{f}})}\left(G_{\sigma}\right), \\
& \forall \mathrm{f} \notin \mathscr{E}_{G(\hat{\mathrm{f}})}(G), \exists \sigma_{0}>0, \forall \sigma>0, \sigma \leq \sigma_{0} \Rightarrow \mathrm{f} \notin \mathscr{E}_{G(\hat{\mathrm{f}})}\left(G_{\sigma}\right) .
\end{aligned}
$$

Using the fact that $\forall \mathrm{f} \in \ell^{2}\left(\mathscr{O}^{d}\right), J_{\sigma}(\mathrm{f}) \leq\|\mathrm{f}\|_{0} \Longrightarrow G_{\sigma}(\mathrm{f}) \leq$ $G(\mathrm{f})$ it comes that $\forall \sigma, \mathscr{E}_{G(\hat{\mathrm{f}})}(G) \subseteq \mathscr{E}_{G(\hat{\mathrm{f}})}\left(G_{\sigma}\right)$ from which (13) is directly deduced.

In order to show condition (14), let $\mathrm{f} \notin \mathscr{E}_{G(\hat{\mathrm{f}})}(G)$ which is equivalent to:

$$
G(\mathrm{f})>G(\hat{\mathrm{f}}) \text {. }
$$

On which condition on $\sigma$ do we have f $\notin \mathscr{E}_{G(\hat{\mathrm{f}})}\left(G_{\sigma}\right)$ ?

$$
\begin{aligned}
\mathrm{f} \notin \mathscr{E}_{G(\hat{\mathrm{f}})}\left(G_{\sigma}\right) \Leftrightarrow & G_{\sigma}(\mathrm{f})>G(\hat{\mathrm{f}}) \\
& \Leftrightarrow J_{d}(\mathrm{f})+\lambda J_{\sigma}(\mathrm{f})>G(\hat{\mathrm{f}}) \\
& \Leftrightarrow J_{d}(\mathrm{f})+\lambda N-\lambda \sum_{(i, j) \in \mathscr{O}^{d}} h_{\sigma}(\mathrm{f}(i, j))>G(\hat{\mathrm{f}}) \\
& \Leftrightarrow \lambda \sum_{(i, j) \in \mathscr{O}^{d}} \exp \left(-\frac{\mathrm{f}_{i j}^{2}}{2 \sigma^{2}}\right)<J_{d}(\mathrm{f})+\lambda N-G(\hat{\mathrm{f}}) .
\end{aligned}
$$


Let $N_{\mathrm{f}}^{0}$ be the number of zeros of f. If $N_{\mathrm{f}}^{0}=N$, (16) is equivalent to $0<J_{d}(\mathrm{f})-G(\hat{\mathrm{f}})=G(\mathrm{f})-G(\hat{\mathrm{f}})$ which is satisfied $\forall \sigma$ since we have inequality (15). If $N_{\mathrm{f}}^{0}<N$, we define $\mathrm{f}_{m}=\min \left\{\left|\mathrm{f}_{i j}\right| \neq 0\right\}_{(i, j) \in \mathscr{O}^{d}}$, then we can write:

$$
\lambda \sum_{(i, j) \in \mathscr{O}^{d}} \exp \left(-\frac{\mathrm{f}_{i j}^{2}}{2 \sigma^{2}}\right) \leq \lambda N_{\mathrm{f}}^{0}+\lambda\left(N-N_{\mathrm{f}}^{0}\right) \exp \left(-\frac{\mathrm{f}_{m}^{2}}{2 \sigma^{2}}\right) .
$$

Thus, $\forall \sigma$ for which:

$$
\lambda N_{\mathrm{f}}^{0}+\lambda\left(N-N_{\mathrm{f}}^{0}\right) \exp \left(-\frac{\mathrm{f}_{m}^{2}}{2 \sigma^{2}}\right)<J_{d}(\mathrm{f})+\lambda N-G(\hat{\mathrm{f}})
$$

is satisfied, (16) will be also satisfied thanks to (17). Let's now working on inequality (18). We can rewrite (18) as follows:

$$
\lambda\left(N-N_{\mathrm{f}}^{0}\right) \exp \left(-\frac{\mathrm{f}_{m}^{2}}{2 \sigma^{2}}\right)<J_{d}(\mathrm{f})+\lambda\left(N-N_{\mathrm{f}}^{0}\right)-G(\hat{\mathrm{f}}) .
$$

Using inequality (15) we have $J_{d}(\mathrm{f})+\lambda\left(N-N_{\mathrm{f}}^{0}\right)=G(\mathrm{f})>G(\hat{\mathrm{f}})$ which implies that the right hand side of (19) is strictly positive. Thus we can make the following development:

$$
\begin{aligned}
& \lambda\left(N-N_{\mathrm{f}}^{0}\right) \exp \left(-\frac{\mathrm{f}_{m}^{2}}{2 \sigma^{2}}\right)<G(\mathrm{f})-G(\hat{\mathrm{f}}) \\
\Leftrightarrow & -\frac{\mathrm{f}_{m}^{2}}{2 \sigma^{2}}<\log \left(\frac{1}{\lambda\left(N-N_{\mathrm{f}}^{0}\right)}[G(\mathrm{f})-G(\hat{\mathrm{f}})]\right) \\
\Leftrightarrow & \frac{1}{\sigma^{2}}>-\frac{2}{\mathrm{f}_{m}^{2}} \log (A)
\end{aligned}
$$

where $A=\frac{1}{\lambda\left(N-N_{\mathrm{f}}^{0}\right)}[G(\mathrm{f})-G(\hat{\mathrm{f}})]$. We can discern two cases:

- If $A \geq 1$ then the right hand side of (20) is negative or zero and so, (20) holds $\forall \sigma$.

- If $A<1$ then the right hand side of (20) is strictly positive and (20) holds only if $\sigma<\mathrm{f}_{m} \log ^{-\frac{1}{2}}\left(\frac{1}{A^{2}}\right)=\sigma_{0}$

Consequently, there exists a $\sigma_{0}>0$ such that inequality (18) holds $\forall \sigma \leq \sigma_{0}$ and, combined with (17), imply (16) which is equivalent to f $\notin \mathscr{E}_{G(\hat{\mathrm{f}})}\left(G_{\sigma}\right)$ and prove (14). Both conditions (13) and (14) are then satisfied that is equivalent to (12) which, with (11) finish the proof.

\section{NUMERICAL RESULTS}

In this section we present some numerical results on synthetic data. We use a simulated membrane (3D) shown on Figure 2. From this synthetic sample, a numerical simulator of the microscope is used in order to get the MA-TIRFM acquisitions (set of 2D images) according to the model in $(\mathcal{P})$. Two images of this set are also presented on Figure 2. These acquisitions are performed using a critical angle equal to $\alpha=61^{\circ}$ and 30 different incident angles from $62^{\circ}$ to $80^{\circ}$. The noise level $b_{g}$ (on the acquired images) is set to $10 \%$ of the maximum intensity of the simulated sample.

Since we are interested in the estimation of the axial membrane position, we quantify the robustness of the method using the position errors along the axial direction as shown on Figure 3. For each pixel in the $(x, y)$ plane we compute the absolute error between the axial membrane positions of the simulated sample and the reconstructed one. This error is quantified using the colormap of the Figure 3 (dark blue $=0 \mathrm{~nm}$ and dark red corresponds to an error $>50 \mathrm{~nm}$ ).
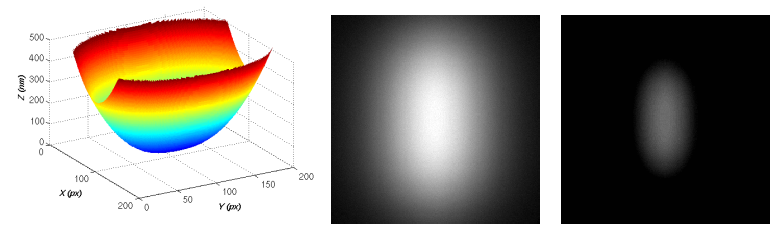

Fig. 2. Left: Synthetic membrane. Right: Microscope acquisition (numerical simulations) with the angles $\alpha=62^{\circ}$ and $\alpha=65.1^{\circ}$.
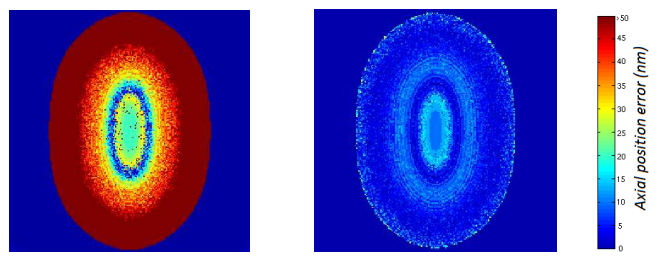

Fig. 3. Position errors of the reconstructed membrane with different algorithms. Left: Richardson-Lucy (without regularization) [14]. Right: Algorithm $1(\lambda=0.001)$.

As we can see on Figure 3 right, we obtain a high accuracy reconstruction with the Algorithm 1 compared to the solution obtained without regularization (Figure 3 left). Indeed the axial position errors are between 5 and $15 \mathrm{~nm}$ for the membrane reconstructed using the $\ell^{0}$ regularization whereas it varies from 30 to more than $50 \mathrm{~nm}$ for the reconstruction without regularization. Following the theoretical TIRFM model $(\mathcal{P})$, it is easy to see that the exponential decay of the evanescent field causes that the deeper the structures, the lower the signal contribution on the acquired images (ill-posedness of the problem) which explain the large errors obtained without regularization (Figure 3 left). We have also tested to reconstruct the membrane using Total Variation (TV) regularization. The reconstruction obtained is similar, in term of axial position errors, to the one presented on the Figure 3 left (without regularization). Finally, using an $\ell^{1}$ regularization, the axial position errors are of the same order of magnitude than the errors showed on Figure 3 right $\left(\ell^{0}\right.$ regularization) for structures below $200 \mathrm{~nm}$ in the axial direction but increase drastically above this limit while the errors with the $\ell^{0}$ regularization remain between 5 and $15 \mathrm{~nm}$ until structures greater than $300 \mathrm{~nm}$. However, due to page limit comparisons with TV and $\ell^{1}$ regularizations are not presented here as well as results on the reconstruction of fine particles.

\section{CONCLUSION}

In this paper we proposed a new model for the MA-TIRFM reconstruction problem based on a $\ell^{0}$ regularization to enforce the sparsity of the solution. Following the idea proposed in [9] for finding sparse solutions of underdetermined system of linear equations, we propose to minimize a sequence of functional $G_{\sigma}$ (Problem $\left(\mathcal{P}_{2}^{\sigma}\right)$ ) which are smooth approximations of the objective function $G$ (Problem $\left.\left(\mathcal{P}_{2}\right)\right)$ and for which, global minimizers when $\sigma$ tends to zero are also global minimizers of $G$ (Theorem 1). Thus if for each $\sigma$ of the sequence, Algorithm 1 does not get trapped into a local minimizer of $G_{\sigma}$, it will converge to a global solution of $\mathcal{P}_{2}$. Finally, simulations results show the accuracy of this approach. Future work will focus on the possibility to construct an optimal sequence $\sigma$ which ensures the convergence to a global solution of $\mathcal{P}_{2}$. 


\section{REFERENCES}

[1] Daniel Axelrod, Edward H Hellen, and Robert M Fulbright, "Total internal reflection fluorescence," in Topics in fluorescence spectrometry, pp. 289-343. Springer, 2002.

[2] GA Truskey, JS Burmeister, E Grapa, and WM Reichert, "Total internal reflection fluorescence microscopy (tirfm). II. topographical mapping of relative cell/substratum separation distances," Journal of cell science, vol. 103, no. 2, pp. 491-499, 1992.

[3] Bence P Ölveczky, N Periasamy, and AS Verkman, "Mapping fluorophore distributions in three dimensions by quantitative multiple angle-total internal reflection fluorescence microscopy.," Biophysical journal, vol. 73, no. 5, pp. 2836, 1997.

[4] Alexander Rohrbach, "Observing secretory granules with a multiangle evanescent wave microscope.," Biophysical journal, vol. 78, no. 5, pp. 2641, 2000.

[5] Dinah Loerke, Walter Stühmer, Martin Oheim, et al., "Quantifying axial secretory-granule motion with variable-angle evanescent-field excitation.," Journal of neuroscience methods, vol. 119, no. 1, pp. 65, 2002.

[6] Qian Yang, Alexander Karpikov, Derek Toomre, and James Duncan, "3D reconstruction of microtubules from multipleangle total internal reflection fluorescence microscopy using Bayesian framework," IEEE transactions on Image Processing, pp. 2248-2259, 2011.

[7] Liang Liang, Hongying Shen, Yingke Xu, Pietro De Camilli, Derek K Toomre, and James S Duncan, "A Bayesian method for 3D estimation of subcellular particle features in multi-angle tirf microscopy," in International Symposium on Biomedical Imaging (ISBI). IEEE, 2012, pp. 984-987.

[8] Emmanuel Soubies, Laure Blanc-Féraud, Sébastien Schaub, and Gilles Aubert, "A 3D model with shape prior information for biological structures reconstruction using Multiple-Angle Total Internal Reflection Fluorescence Microscopy.," in International Symposium on Biomedical Imaging (ISBI). IEEE, 2014.

[9] Hosein Mohimani, Massoud Babaie-Zadeh, and Christian Jutten, "A fast approach for overcomplete sparse decomposition based on smoothed 10 norm," Signal Processing, IEEE Transactions on, vol. 57, no. 1, pp. 289-301, 2009.

[10] Andrew Blake and Andrew Zisserman, Visual reconstruction, vol. 2, MIT press Cambridge, 1987.

[11] Saima Ben Hadj, Laure Blanc-Féraud, and Gilles Aubert, "Space Variant Blind Image Restoration," Research Report RR-8073, INRIA, 2012.

[12] Nicolas Dey, Laure Blanc-Féraud, Christophe Zimmer, Pascal Roux, Zvi Kam, Jean-Christophe Olivo-Marin, and Josiane Zerubia, "Richardson-Lucy algorithm with total variation regularization for 3D confocal microscope deconvolution," $M i$ croscopy research and technique, vol. 69, no. 4, pp. 260-266, 2006.

[13] Kazimierz Kuratowski, Leo F Boron, and Ryszard Engelking, Introduction to set theory and topology, vol. 13, Pergamon Press New York, 1961.

[14] William Hadley Richardson, "Bayesian-based iterative method of image restoration," JOSA, vol. 62, no. 1, pp. 55-59, 1972. 